\title{
Sortilin and SorLA Display Distinct Roles in Processing and Trafficking of Amyloid Precursor Protein
}

\author{
Camilla Gustafsen, Simon Glerup, Lone Tjener Pallesen, Ditte Olsen, Olav M. Andersen, Anders Nykjær, Peder Madsen, \\ and Claus Munck Petersen \\ The Lundbeck Foundation Research Centre MIND, Department of Biomedicine, Aarhus University, DK-8000 Aarhus C, Denmark
}

The development and progression of Alzheimer's disease is linked to excessive production of toxic amyloid- $\beta$ peptide, initiated by $\beta$-secretase cleavage of the amyloid precursor protein (APP). In contrast, soluble $\operatorname{APP} \alpha(\operatorname{sAPP} \alpha)$ generated by the $\alpha$-secretase is known to stimulate dendritic branching and enhance synaptic function. Regulation of APP processing, and the shift from neurotrophic to neurotoxic APP metabolism remains poorly understood, but the cellular localization of APP and its interaction with various receptors is considered important. We here identify sortilin as a novel APP interaction partner. Like the related APP receptor SorLA, sortilin is highly expressed in the CNS, but whereas SorLA mainly colocalizes with APP in the soma, sortilin interacts with APP in neurites. The presence of sortilin promotes $\alpha$-secretase cleavage of APP, unlike SorLA, which inhibits the generation of all soluble products. Also, sortilin and SorLA both bind and mediate internalization of sAPP but to different cellular compartments. The interaction involves the 6A domain of APP, present in both neuronal and non-neuronal APP isoforms. This is important as sAPP receptors described so far only bind the non-neuronal isoforms, leaving SorLA and sortilin as the only receptors for sAPP generated by neurons. Together, our findings establish sortilin, as a novel APP interaction partner that influences both production and cellular uptake of sAPP.

\section{Introduction}

Amyloid precursor protein (APP) is expressed in isoforms, which all undergo sequential cleavage by secretases, resulting in a number of soluble fragments (Haass, 2004). The physiological function of APP and regulation of its proteolysis is not clear, but especially the amyloidogenic pathway of APP processing which leads to production of the soluble fragment sAPP $\beta$ and the aggregation-prone peptide amyloid- $\beta(\mathrm{A} \beta$ ) has received attention for its role in the development of Alzheimer's disease. However, the majority of the cellular APP pool is processed in the non-amyloidogenic pathway by $\alpha$-secretase cleavage resulting in release of sAPP $\alpha$ to the extracellular space. Findings have shown that the soluble forms of APP have a strong neurotrophic effect on cultured neurons (Mattson et al., 1993), regulate the function of membrane-spanning APP in neurite outgrowth (YoungPearse et al., 2008) and reduces neuronal cell loss following diffuse brain injury in rats (Thornton et al., 2006). Furthermore, intraventricular administration of sAPP $\alpha$ improves spatial memory in mice (Bour et al., 2004) and increases the number of presynaptic terminals and memory retention in rats (Roch et al., 1994). A number of receptors influence the metabolism of APP

Received May 15, 2012; revised Sept. 19, 2012; accepted 0ct. 14, 2012.

Author contributions: C.G., S.G., and C.M.P. designed research; C.G., S.G., and L.T.P. performed research; D.O., 0.M.A., A.N., and P.M. contributed unpublished reagents/analytic tools; C.G., S.G., L.T.P., O.M.A., P.M., and C.M.P. analyzed data; C.G. and S.G. wrote the paper.

The Lundbeck Foundation and Riisforts Foundation financially supported this study. We thank Anne Marie Bundsgaard and MIND technicians for skilled assistance.

Correspondence should be addressed to Camilla Gustafsen, MIND Centre, Department of Biomedicine, Ole Worms Alle 3, Bldg 1170, Aarhus University, DK-8000 Aarhus C, Denmark. E-mail: cg@biokemi.au.dk.

DOI:10.1523/JNEUROSCI.2371-12.2013

Copyright $\odot 2013$ the authors $\quad 0270-6474 / 13 / 330064-08 \$ 15.00 / 0$ and the generation of SAPP $\alpha$. Among these are LRP1 and LRP1b, which affect the ratio between $\alpha$ - and $\beta$-secretase processing of APP by altering the internalization rate of membrane-bound APP and mediate endocytosis and degradation of sAPP generated by cleavage of the non-neuronal isoform of APP (Kounnas et al., 1995; Ulery et al., 2000; Cam et al., 2004). Furthermore, two receptors of the Vps10p-domain family, SorLA and SorCS1, both linked to the development of $\mathrm{AD}$, have been shown to modulate APP processing (Andersen et al., 2005; Lane et al., 2010; Fjorback et al., 2012). We here identify the related receptor sortilin as a novel APP interaction partner that modulates APP processing in a manner distinct from SorLA and SorCS1, by promoting the production of sAPP $\alpha$. Sortilin/APP colocalization was detected primarily in neurites of hippocampal neurons in contrast to SorLA/APP, which only colocalize in the soma. We further find that both receptors internalize the neuronal isoform of sAPP, rendering sortilin and SorLA the first described neuronal sAPP endocytosis receptors.

\section{Materials and Methods}

DNA constructs and recombinant proteins. Soluble human APP751 (sAPP751) (NP_958816) and APPd1d2d3KPI (M1-M345) was C-terminally His tagged and expressed in CHO-K1 cells, and purified using Talon beads (Invitrogen). Human sortilin, furin cleavage-resistant immature sortilin (prosortilin), and endocytosis-impaired sortilin (sort mut) have been described previously (Munck Petersen et al., 1999; Nielsen et al., 2001). Sortilin propeptide was expressed as a GST-fusion protein (Munck Petersen et al., 1999). APP695 and APP fragments were produced as described by Andersen et al. (2006). Neurotensin was from Sigma-Aldrich.

Transfected cell lines. HEK293 and CHO-K1 cells were cultured and transfected as described by Nielsen et al. (2007). Stably transfected clones were maintained at $100 \mu \mathrm{g} / \mathrm{ml}$ zeocin (Invitrogen). 
Primary cultures. Hippocampal neurons and cortical astrocytes were prepared from C57BL/6J, Sort $1^{-1-}$ (Jansen et al., 2007), and Sorl1 ${ }^{-1-}$ (Andersen et al., 2005) postnatal day 0 pups. For neuron cultures, tissue was digested with papain $(20 \mathrm{U} / \mathrm{ml})$ and seeded in poly-L-lysine/laminincoated dishes or coverslips in Neurobasal medium (Invitrogen) supplemented with B27 (Invitrogen), Glutamax (Invitrogen), primocin (Lonza) and $20 \mu \mathrm{M} 5$-fluoro-2'-deoxyuridine/uridine, and maintained at $37^{\circ} \mathrm{C}, 5 \% \mathrm{CO}_{2}$ for $8-12 \mathrm{~d}$ before experiments. For astrocyte cultures, cortices were isolated, digested in $0.25 \%$ trypsin (Invitrogen) $/ 0.6 \mathrm{~mm}$ EDTA solution, and seeded into poly-L-lysine-coated T75 flask. Cells were maintained in DMEM with 10\% FBS and primocin until confluency. Flasks were then shaken at $250 \mathrm{rpm}$ for $5 \mathrm{~h}$ and medium with less adherent microglial cells was aspirated, before lysis with $1 \%$ Triton $\mathrm{X}-100$.

Coimmunoprecipitation. Cells were incubated with $5 \mathrm{~mm}$ dithiobis(succinimidyl propionate) (Pierce) for $30 \mathrm{~min}$ at room temperature before addition of $1 \mathrm{~m}$ Tris and lysis of cells with $1 \%$ Triton X-100. Protein complexes were immunoprecipitated using GammaBind beads (GE Healthcare).

Western blotting. Western blot analysis were performed using precast NuPAGE 4-12\% Bis-Tris, Nitrocellulose iBlot Gel Transfer Stacks (Invitrogen) and the following antibodies; anti-sortilin $\mathrm{mAb}$ (Transduction Laboratories), anti-sAPP $\alpha / \mathrm{WO} 2$, anti-sAPP $\beta$ (IBL International), antiAPP/1227 (APP C-terminal), anti-SorLA/20C11, anti-GAPDH/G8795 and anti- $\beta$-actin/5441 (Sigma), anti-Golgi 58K protein/ab6284 (Abcam). Proteins were visualized with HRP-conjugated secondary antibodies (Cell Signaling Technology) and ECL substrate (Pierce).

Immunofluorescence. Cells were fixed in $4 \%$ paraformaldehyde and permeabilized using $0.1 \%$ Triton $\mathrm{X}-100$ before incubation with primary antibodies; anti-sortilin/F11 and anti-SorLA/20C11, anti-sortilin/ AF2934 and anti-APP/AF1168 (R\&D Systems), anti-TGN46/AHP500 (Adb Serotec), anti-LAMP1/ab24170 (Abcam), anti-neurofilament 200/ N4142 (Sigma) and fluorescent secondary antibodies (Invitrogen). Nuclei were visualized with Hoechst dye (Sigma Aldrich). Images were acquired on a Zeiss 710 confocal microscope.

Proximity ligation assay. The proximity ligation assay (PLA) (DuolinkII) was performed according to the manufacturer's protocol with the primary antibodies anti-sortilin/F11, anti-SorLA/20C11, and anti-APP/AF1168, followed by incubation with secondary antibodies conjugated to oligonucleotides, which hybridize to subsequently added circle-forming oligonucleotides and prime a rolling circle amplification, if the antigens are located within proximity of $40 \mathrm{~nm}$. The amplified DNA was visualized by addition of complementary fluorescent-labeled oligonucleotides.

Surface plasmon resonance. Binding of APP fragments to immobilized sortilin was detected by surface plasmon resonance (SPR) using a Biacore 3000 instrument (Munck Petersen et al., 1999).

APP processing. HEK293 cells were seeded (750,000/6-well). After $48 \mathrm{~h}$, conditioned medium was collected and cells were lysed in $1 \%$ Triton X-100. Sortilin, SorLA, GAPDH, APP and sAPP fragments were detected by Western blotting. A $\beta 40$ was measured by ELISA (Sigma), but was below detection limit. Hippocampal neurons were seeded (250,000/12well), and maintained in culture. Medium was conditioned for $48 \mathrm{~h}$ from DIV 8 to 10 , and the concentration of $\operatorname{sAPP} \alpha$ was assessed (Mouse/Rat sAPP $\alpha$ Assay Kit, IBL International). The protein concentration in lysates was determined using Bicinchoninic Acid Kit (Sigma). Significance was evaluated using two-tailed $t$ test.

Subcellular fractionation. Preparation of postnuclear supernatant from transfected cell-lines and subsequent subcellular fractionation by Optiprep gradient centrifugation was performed as previously described (Andersen et al., 2005). For subcellular fractionation of whole brain homogenates brains ( $n=3$, age 16 weeks) were pooled, homogenized in ice-cold Solution A [containing, in mm: 0.25 sucrose, 1 EDTA, 20 HEPES-KOH, pH 7.4 plus Complete (Roche)], and centrifuged for 10 $\min$ at $1000 \times g \rightarrow$ supernatants were centrifuged for 10 min at $3000 \times$ $g \rightarrow$ supernatants were centrifuged for $10 \mathrm{~min}$ at 13,000 rpm (Ti70.1 rotor) $\rightarrow$ supernatants were centrifuged for $45 \mathrm{~min}$ at 50,000 rpm. Resulting pellets were dissolved in Solution A and layered on a continuous $0.8-1.6 \mathrm{~m}$ sucrose- $1 \mathrm{~m}$ KOH gradient prepared using a BioComp gradient master, and centrifuged at 25,000 rpm for $18 \mathrm{~h}$ (SW41 rotor) before collection of 24 fractions.

Internalization of SAPP. Soluble APP751 was labeled using Alexa Fluor 488 Microscale Protein Labeling Kit (Invitrogen) according to the manufacturer's protocol. Cells were incubated with $20 \mathrm{nM} \mathrm{sAPP}$ for $40 \mathrm{~min}$ at $37^{\circ} \mathrm{C}$ before fixation and immunofluorescence staining for receptor expression, TGN46 and LAMP1. For inhibition of lysosomal degradation, cells were treated with leupeptin $(100 \mu \mathrm{g} / \mathrm{ml}) /$ pepstatin $(50 \mu \mathrm{g} / \mathrm{ml}) 24 \mathrm{~h}$ (incubation medium was changed every $6 \mathrm{~h}$ ) before and during the experiment. Hippocampal neurons (DIV12) were incubated with $20 \mathrm{~nm}$ sAPP mixed with $3 \mu \mathrm{M}$ GST-propeptide or GST for $15 \mathrm{~min}$. The number of vesicles containing fluorescent SAPP was quantified from $z$-stacked images of at least 30 randomly selected cells on four different coverslips for each condition using the Spot detection module of Imaris 7.4.2 image analysis software (Bitplane) with a fixed intensity threshold. Colocalization was quantified in a similar manner using the Coloc module of Imaris. Significance was assessed using two-tailed $t$ test.

Quantitative reverse transcriptase PCR. RNA was extracted from brains of C57BL/6J mice ( $n=4$, male, age 12 weeks) followed by reverse transcriptase (RT)-PCR analysis of Actb, Sort1, Sorl1, Lrp1, and Lrp2 (AROS Applied Biotechnology). Receptor expression was normalized to Actb levels as described by Vaerman et al. (2004).

\section{Results}

\section{Sortilin interacts with APP in neuronal processes}

Using quantitative RT-PCR, we found that sortilin is expressed at high levels in the CNS compared with known APP binding receptors (Fig. 1A) and Western blot analysis of primary cultures of astrocytes and hippocampal neurons (Fig. 1B) further suggested that sortilin is mainly found in neurons. To test for an interaction between neuronal APP and sortilin, APP was precipitated from lysate of primary hippocampal neurons with an antibody raised against the intracellular domain of APP. The precipitate was subsequently analyzed by Western blotting following separation by reducing SDS-PAGE. As apparent from Figure $1 C$, sortilin was pulled down by anti-APP but not by control IgG, suggesting the existence of a physical complex between sortilin and membrane spanning APP in neurons.

Immunofluorescent labeling of APP, sortilin and SorLA in hippocampal neurons (Fig. 1D) showed that while APP and sortilin are localized in both neurites and cell body, SorLA is primarily found in the cell soma. To further analyze the colocalization of APP with sortilin and SorLA, we performed a PLA. PLA staining of hippocampal neurons with anti-sortilin and anti-APP antibodies resulted in PLA signals, signifying colocalization, in the neurites of wild-type (WT) neurons, whereas staining with antiSorLA and anti-APP only gave PLA signals in the cell body (Fig. $1 E)$. No PLA signals were detected in receptor deficient neurons obtained from Sort $1^{-/-}$and Sorl $1^{-/-}$mice. Together, these results suggest that sortilin and SorLA colocalize and interact with APP at different neuronal compartments, indicating distinct functional roles of the receptors in relation to APP.

\section{Sortilin interacts with the extracellular 6A domain of APP via a pH-sensitive binding}

APP is expressed in several cell type-specific isoforms. APP695 is the major isoform in neurons, and is distinct from non-neuronal isoforms, such as APP751, by lacking a Kunitz protease inhibitor (KPI) domain (Fig. 2A). Using SPR analysis, we found that immobilized soluble sortilin binds both sAPP695 $\beta$ (Fig. $2 B$ ) and the APP751 ectodomain (sAPP751) (Fig. 2C) in a concentrationdependent manner with dissociation constants in the range of 5-10 nM. Mapping of the domain responsible for the interaction showed that the APP N-terminal domains $\mathrm{d} 1$ and $\mathrm{d} 2$ do not bind to sortilin, whereas binding was observed to a fragment com- 
A

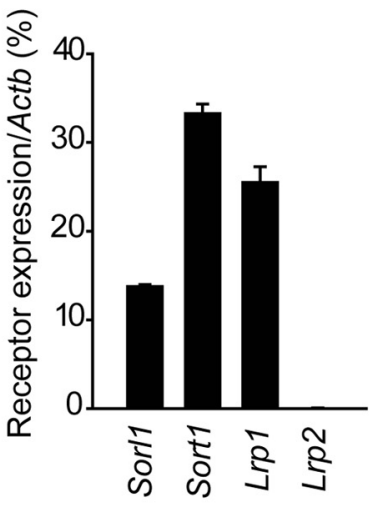

D
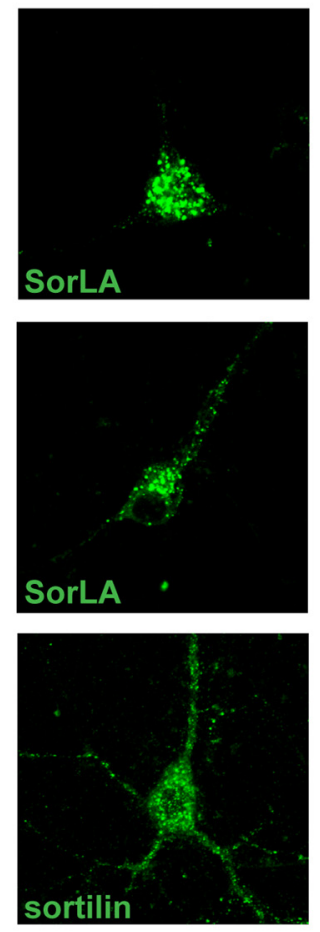

$\mathbf{E}$

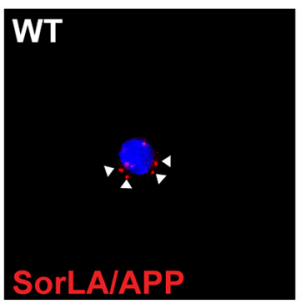

WT

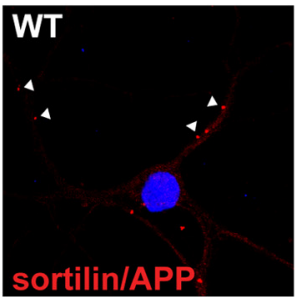

B
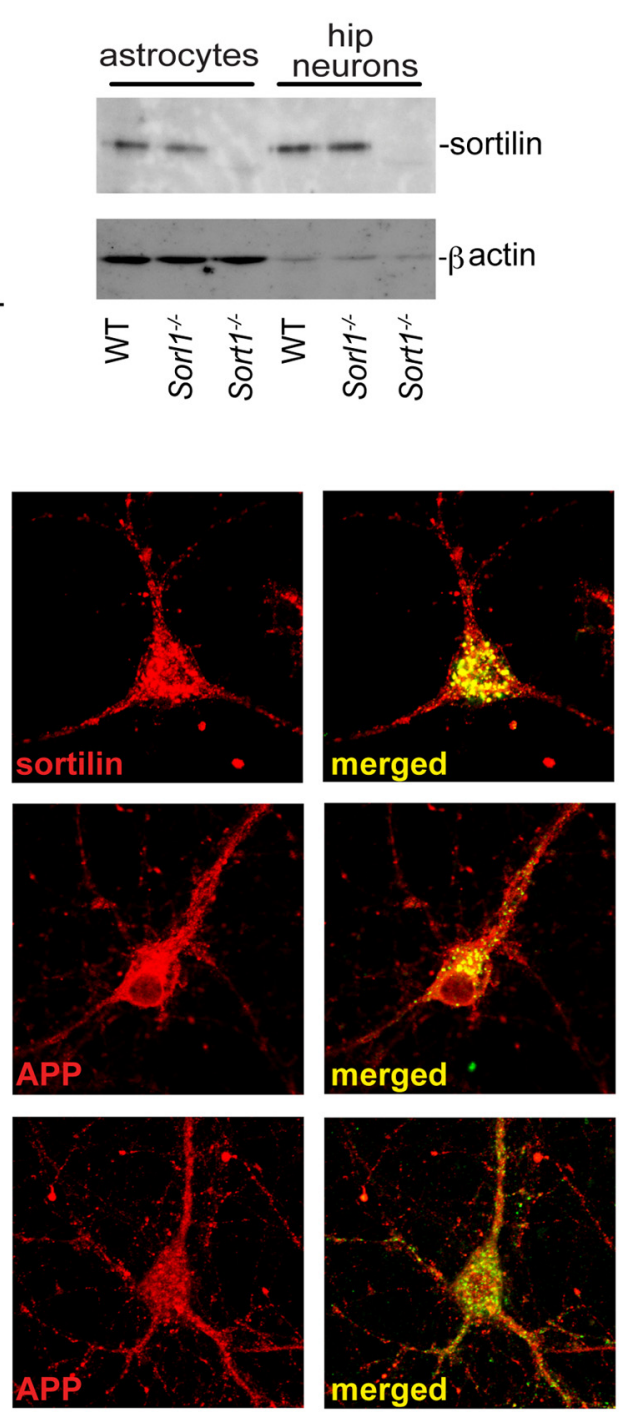

merged
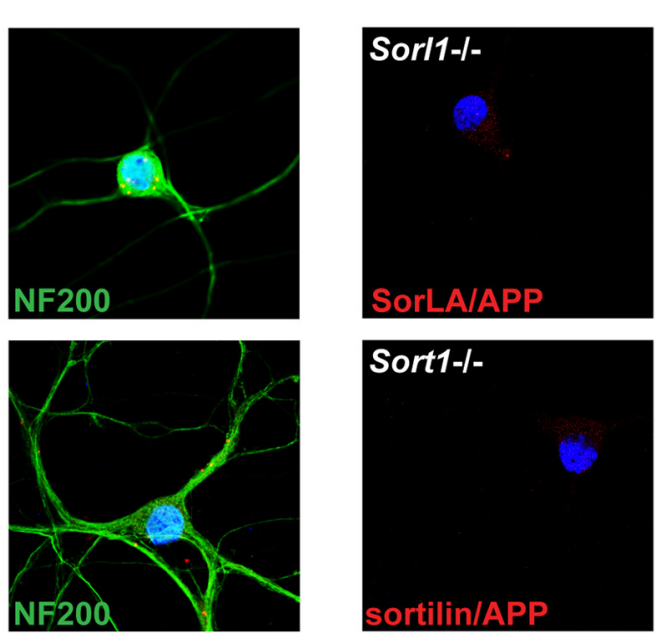

C

IP:

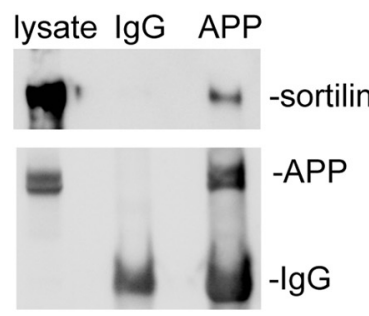

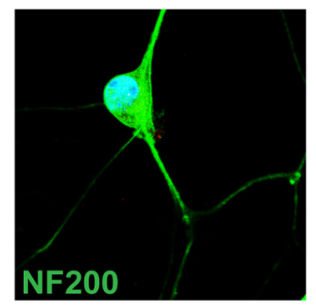

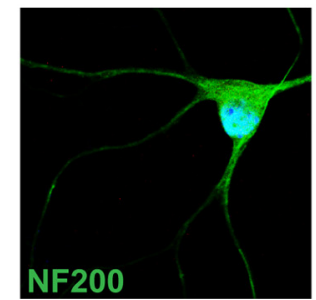

Figure 1. Sortilin is highly expressed in the CNS and colocalize with APP in neurites of hippocampal neurons. A, Quantitative RT-PCR analysis of the receptor genes Sort1, Sorl1, Lrp1, and Lrp2. Expression was normalized to $A c t b$ ( $\beta$-actin) levels (mean values with SDs, $n=4$ mice). $\boldsymbol{B}$, Western blot (WB) analysis of sortilin in astrocytes and neurons obtained from WT, Sorl1 ${ }^{-1-}$, and Sort $^{-1-}$ mice. Note the difference in protein concentration between astrocyte and neuron lysates. C, Immunoprecipitation (IP) of sortilin and APP from hippocampal (Figure legend continues.) 
A

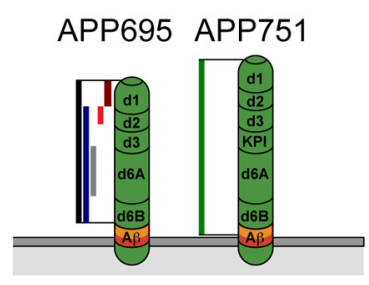

D

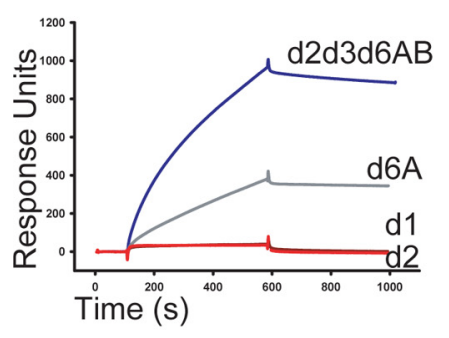

B

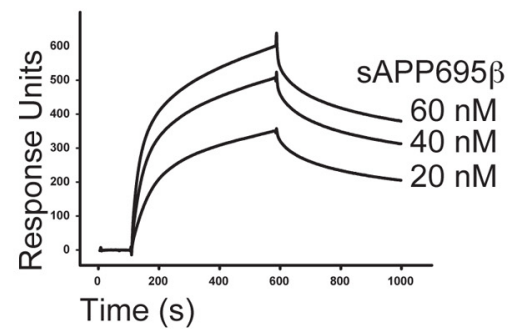

E

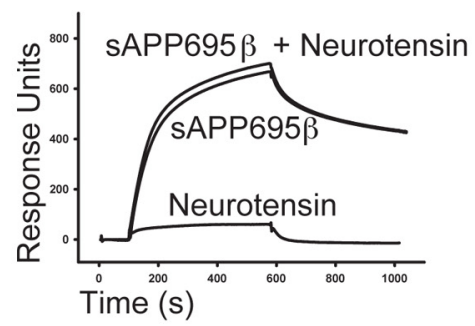

C

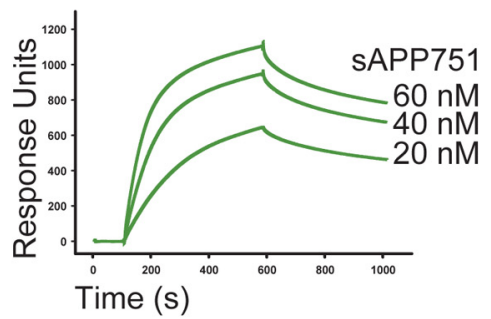

$\mathbf{F}$

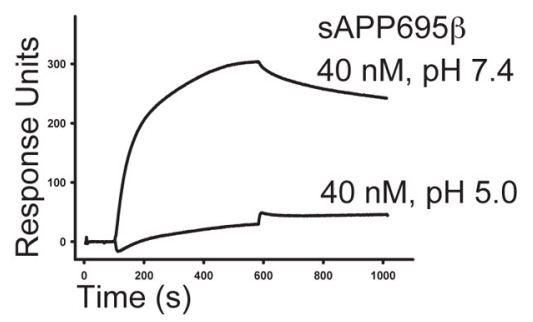

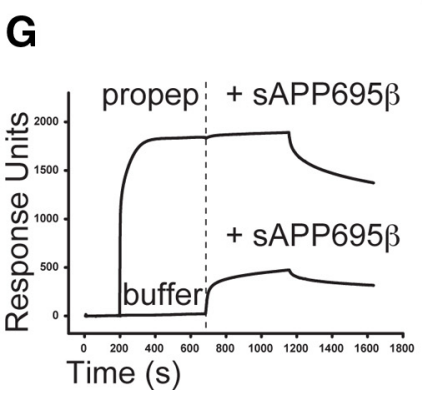
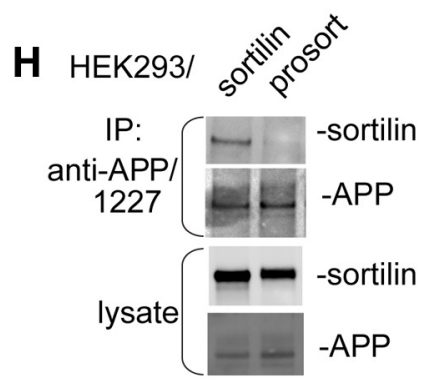

Figure 2. Mature sortilin interacts with APP isoforms 695 and 751 at neutral pH. $\boldsymbol{A}$, The domain structure of APP isoforms 695 and 751 depicted with color code. $\boldsymbol{B}-\mathbf{G}$, SPR analysis of the interaction between APP fragments and immobilized sortilin (extracellular domain) demonstrating binding of sAPP695 $\beta(B)$ and sAPP751 ( () at concentrations of 20 - $60 \mathrm{~nm}$, and APP fragments $(60 \mathrm{~nm})$ containing the domain $6 \mathrm{~A}(\mathrm{~d} 6 \mathrm{~A})(\boldsymbol{D})$. The interaction between sAPP695 $\beta(40 \mathrm{~nm})$ and sortilin was not by inhibited by neurotensin $(\boldsymbol{E})$, but by acidic $\mathrm{pH}(\boldsymbol{F})$. In addition, the interaction was inhibited by GST-sortilin propeptide (propep) binding to sortilin before addition of SAPP695 $\beta$ (indicated by a dotted line; G). H, Co-IP analysis of APP and sortilin from HEK293 cells transfected with sortilin or prosortilin. Precipitated proteins were analyzed by WB. Please note the difference in molecular weight between sortilin and prosortilin.

posed of $\mathrm{d} 2 \mathrm{~d} 3 \mathrm{~d} 6 \mathrm{AB}$ and a fragment covering only the domain $6 \mathrm{~A}$ with an affinity comparable to that of full-length sAPPs (Fig. 2D). A fragment composed of $\mathrm{d} 1 \mathrm{~d} 2 \mathrm{~d} 3$ and the KPI domain bound to sortilin with a dissociation constant of $2 \mu \mathrm{M}$ (data not shown), suggesting that KPI is not critical for sAPP binding. Thus, unlike LRP, sortilin binds both neuronal and non-neuronal APP. Binding was not inhibited by the small peptide ligand neurotensin (Fig. 2E) known to bind in the tunnel cavity of the Vps10p domain (Quistgaard et al., 2009), but the interaction was inhibited by acidic $\mathrm{pH}$ (Fig. $2 F$ ). In addition, the binding was completely inhibited by saturation of sortilin with a GST-fusion protein containing the sortilin propeptide (Fig. 2G). This inhibition was confirmed in a coimmunoprecipitation experiment in which sortilin, but not a sortilin variant with mutated propeptide processing site (prosortilin), was pulled down together with membrane-

\section{$\leftarrow$}

(Figure legend continued.) neurons. Unspecific lgG was used as control. Precipitated proteins were analyzed by WB. D, Immunofluorescence staining of sortilin, SorLA, and APP in WT hippocampal neurons. E, PLA analysis of WT hippocampal neurons followed by immunostaining for neurofilament 200 (NF200). Red spots (marked with white arrowheads) indicate colocalization of APP with sortilin and SorLA, respectively. Sort $1^{-/-}$and Sorl $1^{-/-}$neurons are included as negative controls. spanning APP (Fig. 2H), showing that the interaction with APP requires propeptide cleavage of sortilin.

\section{Sortilin pushes the metabolism of APP toward production of $\operatorname{sAPP} \alpha$}

Sortilin can mediate trafficking from the secretory pathway to endosomes, as well as retrograde transport to the trans-Golgi network (TGN) after internalization from the cell surface (Nielsen et al., 2001). Analysis of the effect of sortilin on the subcellular localization of APP was performed by gradient centrifugation of HEK293 cells transfected with sortilin or sort mut (Fig. 3A) and whole brain homogenates from WT and Sort $1^{-1-}$ mice (Fig. $3 B$ ). Neither overexpression of plasma membraneassociated sort mut nor sortilin deficiency caused a dramatic change in the distribution of APP, suggesting that the intracellular localization of APP is independent of sortilin. However, we found that conditioned medium obtained from sortilintransfected cells contain a higher level of sAPP $\alpha$ and a lower level of sAPP $\beta$ compared with medium from untransfected cells (Fig. $3 C)$. In contrast, cells transfected with SorLA produced lower levels of both sAPP forms (Fig. 3C). In addition, conditioned medium from cultured hippocampal neurons prepared from Sort $1^{-/-}$mice contained a significantly lower level of $\operatorname{sAPP} \alpha$ compared with medium obtained from WT neurons, suggesting 
that sortilin impacts APP proteolysis, favoring $\alpha$-secretase processing.

\section{Sortilin and SorLA sort internalized sAPP to different cellular compartments}

Sortilin and SorLA both mediate endocytosis of ligands bound at the cell surface, and sortilin has been found to facilitate lysosomal degradation of internalized ligands (Nielsen et al., 2001, 2007; Hu et al., 2010). We therefore analyzed receptormediated endocytosis of sAPP in HEK293 cells. Strikingly, cells transfected with sortilin or SorLA showed increased uptake of fluorescence-labeled sAPP compared with untransfected cells and cells transfected with prosortilin (Fig. 4A), showing that both receptors mediate internalization of sAPP. However, sAPP internalized by sortilin appeared in vesicular structures dispersed throughout the cell and showed $56 \pm 13 \%$ colocalization with sortilin. In contrast, $84 \pm 3.5 \%$ colocalization between sAPP and SorLA was observed and internalized sAPP was located in the paranuclear region in structures partially colocalizing with TGN46positive compartments (Fig. 4B). The internalization by SorLA appeared to give a more intense sAPP staining compared with the sAPP uptake of sortilin. Interestingly, we observed $47 \pm 12 \%$ colocalization between sAPP and the lysosomal marker LAMP-1 in sortilin cells (Fig. 4C) compared with $1.8 \pm 1.3 \%$ in SorLAtransfected cells (Fig. 4C), and treatment of cells with lysosomal protease inhibitors before sAPP uptake resulted in a $>3$-fold increase in the number of sAPP-containing vesicles in sortilin-expressing cells, but had no effect on the accumulation of sAPP in SorLA cells (Fig. 4D), indicating that sortilin targets sAPP for lysosomal degradation. These findings are consistent with a $\mathrm{pH}$-sensitive interaction between the two proteins, likely resulting in dissociation after internalization to endosomal compartments of lower $\mathrm{pH}$. We speculated that endogenous sortilin might mediate sAPP endocytosis in neuronal processes. Accordingly, we found that the uptake of sAPP in neurites of hippocampal neurons was markedly reduced by coincubation with excess receptor propeptide (Fig. 4E).

\section{Discussion}

The function and maintenance of neuronal circuitries is based on intricately balanced neurotrophic signals during constant exposure to cellular stressors. The molecular network of APP represents a system that shifts from displaying neurotrophic activity to devastating neuronal toxicity during the progression of

A

anti-APP/1227

anti-Golgi protein $58 \mathrm{~K}$

C
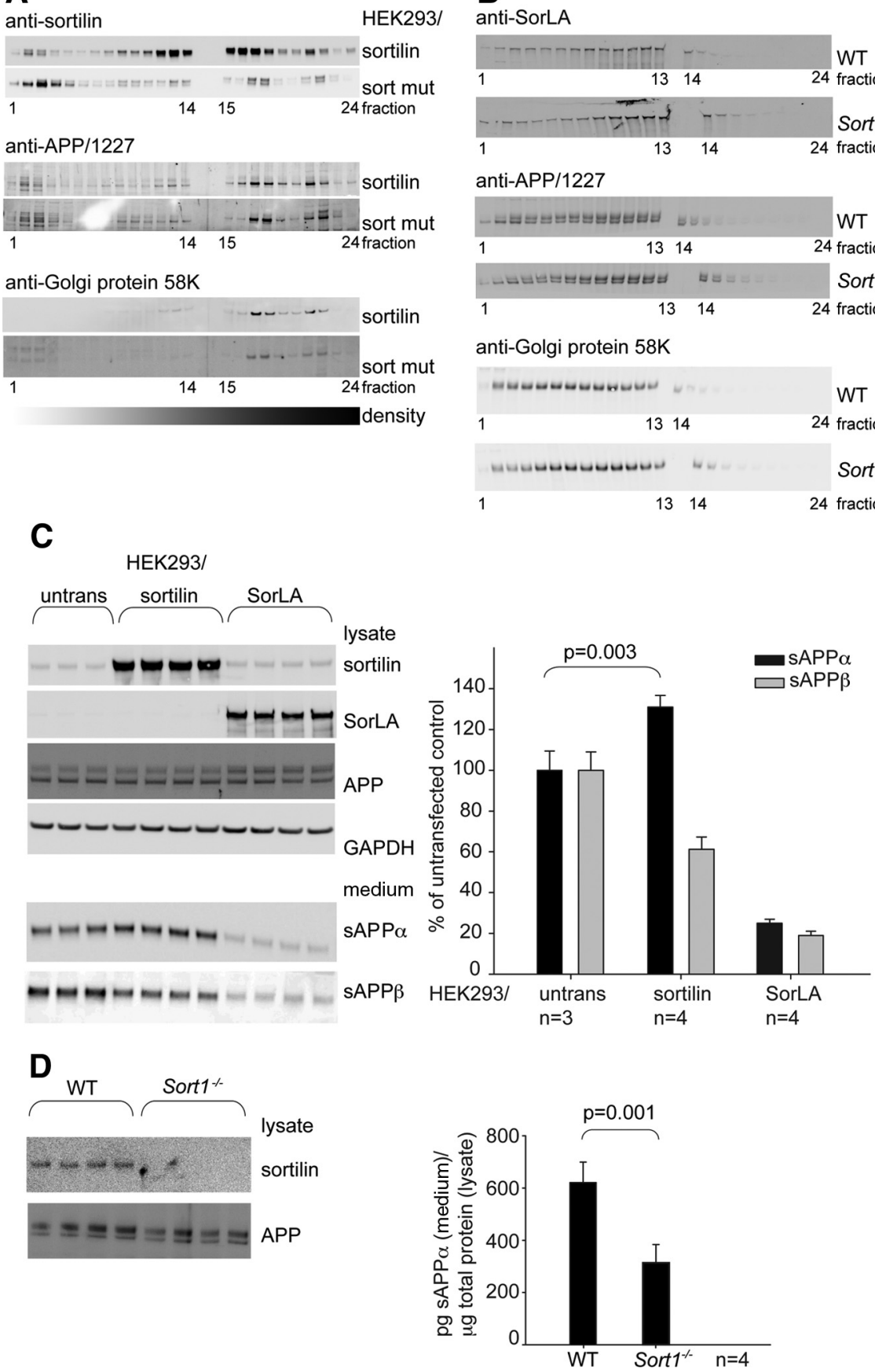

Figure 3. Sortilin pushes APP toward production of SAPP $\alpha$. A, Subcellular fractionation by Optiprep gradient centrifugation of HEK293 cells stably transfected with sortilin or sort mut. WB analysis of sortilin, endogenous APP, and Golgi protein 58K in fractions 1-24. $\boldsymbol{B}$, Subcellular distribution of APP in whole brain homogenates from WT and Sort $1^{-1-}$ mice assess by continuous sucrose gradient centrifugation. WB analysis of SorLA, APP, and Golgi protein 58K in fractions 1-24. C, The APP metabolism in HEK293 cells stably transfected as indicated was analyzed by WB detection of SAPP $\alpha$ and sAPP $\beta$ in $48 \mathrm{~h}$ conditioned medium. Bands were quantified by densitometry and bar graphs show mean values ( $n$ as indicated) with SD of the SAPP levels in percentage of untransfected control. The expression of sortilin, SorLA, APP, and GAPDH in the corresponding lysates is shown. $\boldsymbol{D}$, The level of $\operatorname{SAPP} \alpha$ in conditioned medium of hippocampal neurons obtained from WT and Sort ${ }^{-1-}$ mice was analyzed by ELISA. Bar graphs show mean values with SD $(n=4)$ of pg sAPP $\alpha / \mu \mathrm{g}$ total protein in the corresponding lysate. WBs show expression of APP and sortilin in lysates.
anti-SorLA

anti-APP/1227

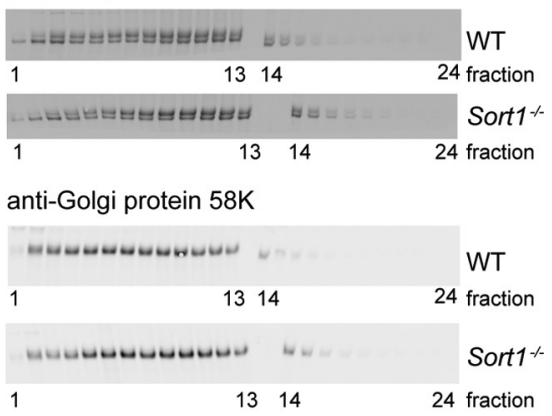

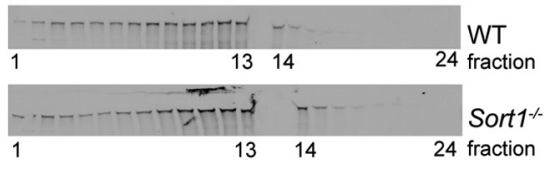


A

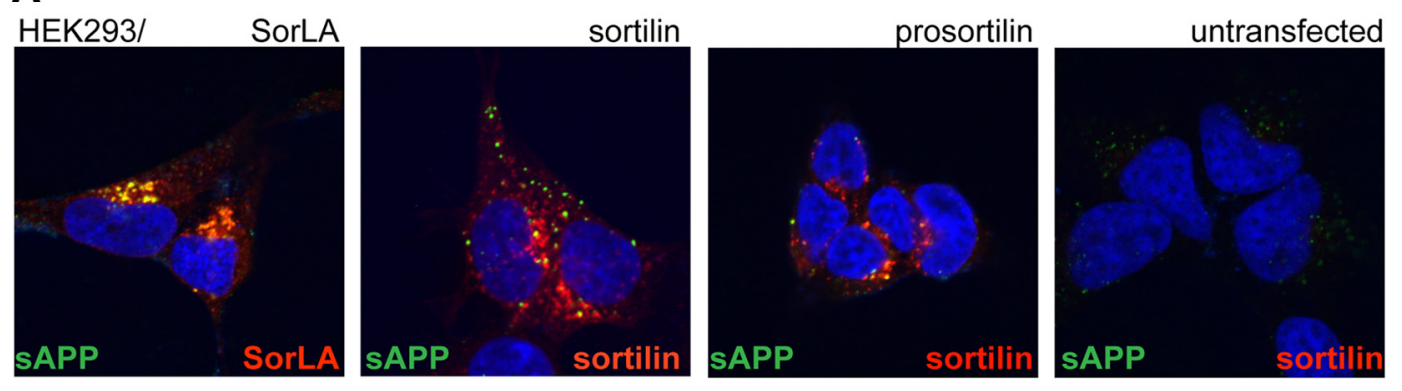

B

C

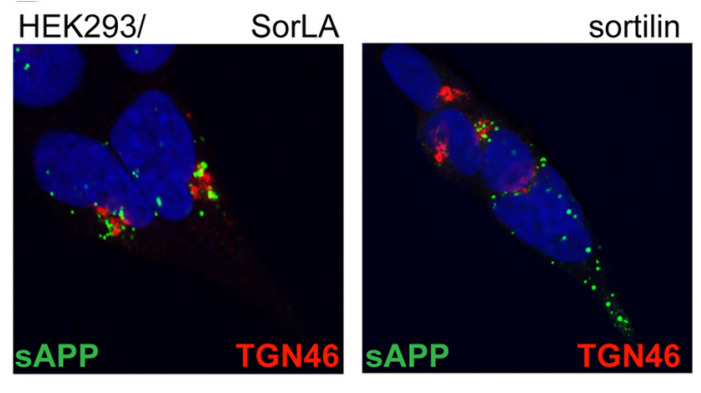

HEK293/ SorLA

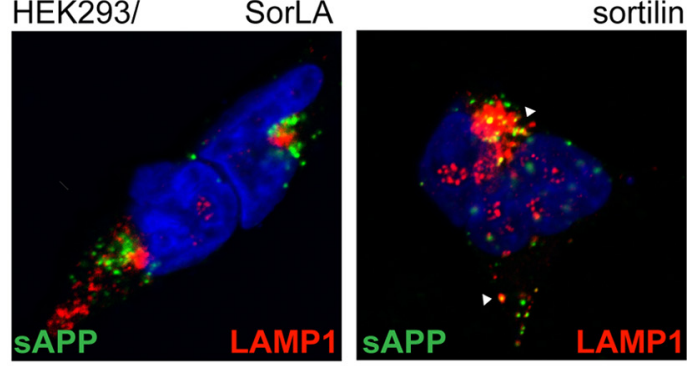

D
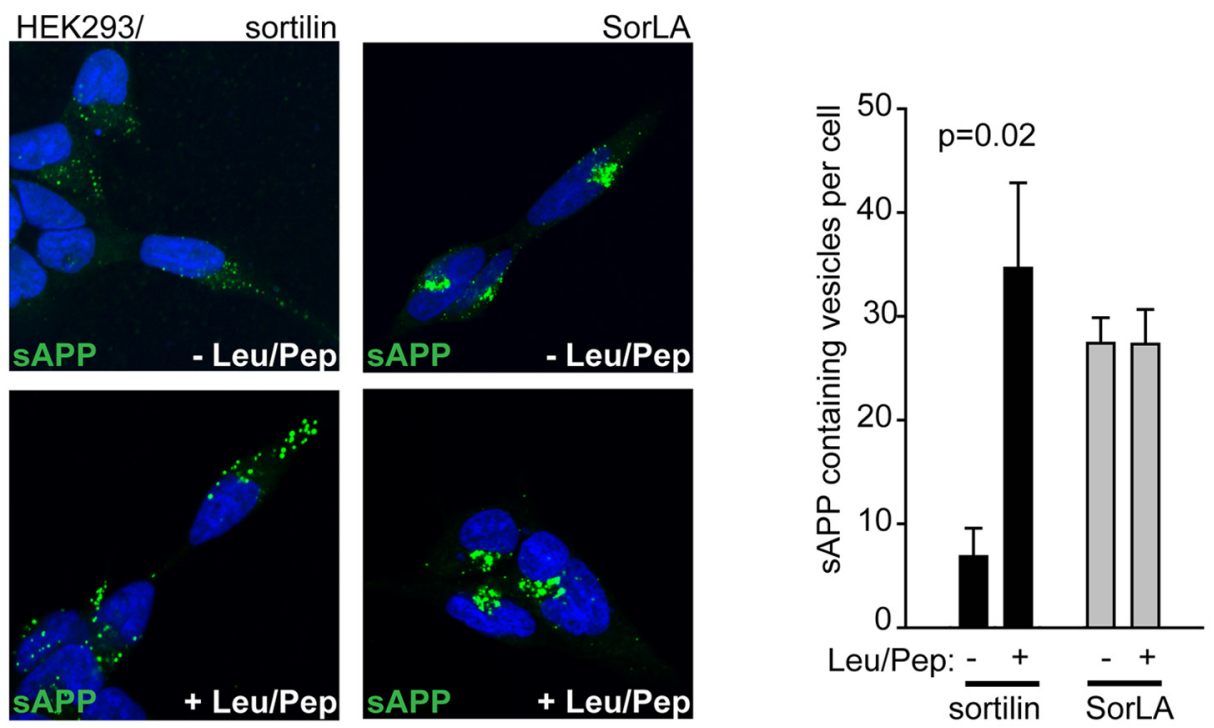

E

WT hippocampal neurons
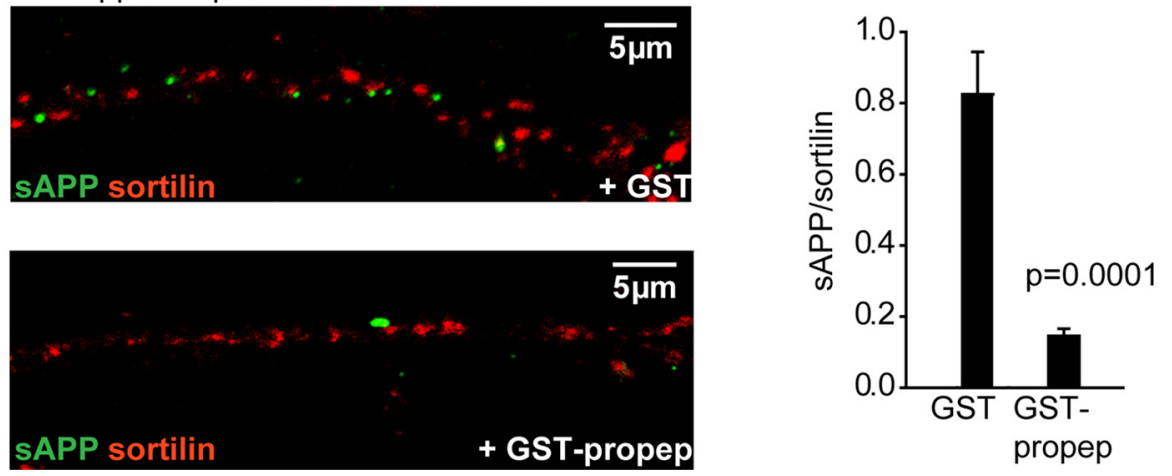

Figure 4. Sortilin and SorLA direct sAPP to distinct cellular compartments. A-C, HEK293 cells, transfected as indicated, were incubated with 20 nm Alexa Fluor 488 -labeled sAPP751 for 40 min at $37^{\circ}$ C. Cells were subsequently stained with anti-sortilin/F11 or anti-SorLA/20C11 antibodies $(\boldsymbol{A})$, anti-TGN46 ( $\left.\boldsymbol{B}\right)$, or anti-LAMP1 (C; arrowheads indicate colocalization). $\boldsymbol{D}$, Uptake of sAPP in sortilin or SorLA-transfected cells pretreated with lysosomal protease inhibitors leupeptin/pepstatin (Leu/Pep). Number of sAPP-containing vesicles were quantified and mean values (Figure legend continues.) 
SorLA. Interestingly, we find that SorLA and sortilin differentially impact processing and sorting of APP. Our results show that SorLA mainly colocalizes with APP in the soma of hippocampal neurons, whereas sortilin colocalizes with APP in neurites. In contrast to SorLA, we find that sortilin shifts the processing toward production of $\operatorname{sAP} \alpha$, possible by optimizing APP as substrate for $\alpha$-secretase cleavage. A recent study reports that sortilin overexpression in HEK293 enhances $\beta$-secretase activity by conducting intracellular trafficking of the enzyme (Finan et al., 2011). However, this apparent discrepancy may be caused by their use of a C-terminally tagged sortilin variant, as we have recently shown that such tagging disturbs the binding of the GGA adaptors (Cramer et al., 2010), and can be expected to affect the subcellular localization of sortilin. Despite these opposing observations in HEK293 cells, hippocampal neurons lacking sortilin display decreased production of $\operatorname{sAPP} \alpha$, suggesting that sortilin promotes $\alpha$-secretase activity in neuronal systems. Hence, sortilin might selectively increase $\alpha$-secretase cleavage in the neuronal processes, hereby displaying a fundamentally different function from that of SorLA, which protects APP against proteolytic processing in compartments localized in the cell body. We further observe that both receptors mediate cellular uptake of soluble APP products. In HEK293 cells, SorLA-mediated internalization of sAPP results in colocalization of receptor and ligand in the paranuclear regions, whereas sortilin facilitates lysosomal degradation of SAPP, as observed for other ligands, e.g., progranulin (Hu et al., 2010). Intriguingly, we find that uptake of sAPP in neurites of hippocampal neurons can be inhibited by addition of receptor propeptide, which blocks binding of sAPP to sortilin (Fig. 2G,H), but not to SorLA (Andersen et al., 2006). Previously, LRP1 and LRP1b have been proposed as clearance receptors for sAPP. The binding of these receptors to sAPP relies on the KPI domain, and the scavenging function is there limited to sAPP produced from KPI-containing APP isoforms (Kounnas et al., 1995; Cam et al., 2004). The KPI-containing isoforms of APP is found in most tissues, and have been reported expressed in the CNS by non-neuronal cells including astrocytes and microglia (Rohan de Silva et al., 1997). This is, however, questioned by a recent study which failed to detect expression of APP in glial cells (Guo et al., 2012), and APP695 is, with its high expression in neurons, the predominating isoform in the CNS. Sortilin and SorLA bind APP independently of the KPI domain, and are to the best of our knowledge, the first identified CNS endocytosis receptors for sAPP produced by cleavage of APP695. Our observations indicate that the consequence of sAPP internalization is receptor dependent, suggesting that the relative levels of sortilin and SorLA at the cell surface are determinative for the cellular fate of sAPP. Although the predominating sortilin effect in hippocampal neurons is on processing, as sortilin deficiency causes a significant decrease in the level of $\operatorname{sAPP} \alpha$, it is likely that endocytosis may dominate in other cellular systems. The combined effect of sortilin and SorLA on APP metabolism is obviously complex and we speculate that the Vps10p-domain receptors represent an APP receptor system that, by compartment-specific interactions and functions, collaborates in the regulation of APP metabolism to

$\leftarrow$

(Figure legend continued.) with SEM is shown as bar graphs, $n=30$ cells/condition. $\boldsymbol{E}$, The uptake of sAPP (20 nM) in WT hippocampal neurites with addition of receptor propeptide fused to GST $(3 \mu \mathrm{m})$ or GST alone was inspected in at least 100 cells per coverslip from eight coverslips from two independent cultures. The number of SAPP-positive vesicles in randomly selected neurites was quantified and normalized to the number of sortilin-positive vesicles, and is shown as bar graphs of mean values. Error bars indicate SEM. promote non-amyloidogenic processing. Further studies are required to elucidate the roles of sortilin and SorLA in the neurotrophic activity of sAPP. Our findings illustrate the complexity of APP metabolism and shows that it is a highly dynamic process that depends on receptor identity and expression levels, and hereby can vary with cell type, brain region, or age.

\section{References}

Andersen OM, Reiche J, Schmidt V, Gotthardt M, Spoelgen R, Behlke J, von Arnim CA, Breiderhoff T, Jansen P, Wu X, Bales KR, Cappai R, Masters CL, Gliemann J, Mufson EJ, Hyman BT, Paul SM, Nykjaer A, Willnow TE (2005) Neuronal sorting protein-related receptor sorLA/LR11 regulates processing of the amyloid precursor protein. Proc Natl Acad Sci U S A 102:13461-13466. CrossRef Medline

Andersen OM, Schmidt V, Spoelgen R, Gliemann J, Behlke J, Galatis D, McKinstry WJ, Parker MW, Masters CL, Hyman BT, Cappai R, Willnow TE (2006) Molecular dissection of the interaction between amyloid precursor protein and its neuronal trafficking receptor SorLA/LR11. Biochemistry 45:2618-2628. CrossRef Medline

Bour A, Little S, Dodart JC, Kelche C, Mathis C (2004) A secreted form of the beta-amyloid precursor protein (sAPP695) improves spatial recognition memory in OF1 mice. Neurobiol Learn Mem 81:27-38. CrossRef Medline

Cam JA, Zerbinatti CV, Knisely JM, Hecimovic S, Li Y, Bu G (2004) The low density lipoprotein receptor-related protein $1 \mathrm{~B}$ retains beta-amyloid precursor protein at the cell surface and reduces amyloid-beta peptide production. J Biol Chem 279:29639-29646. CrossRef Medline

Cramer JF, Gustafsen C, Behrens MA, Oliveira CL, Pedersen JS, Madsen P, Petersen CM, Thirup SS (2010) GGA autoinhibition revisited. Traffic 11:259-273. CrossRef Medline

Finan GM, Okada H, Kim TW (2011) BACE1 retrograde trafficking is uniquely regulated by the cytoplasmic domain of sortilin. J Biol Chem 286:12602-12616. CrossRef Medline

Fjorback AW, Seaman M, Gustafsen C, Mehmedbasic A, Gokool S, Wu C, Militz D, Schmidt V, Madsen P, Nyengaard JR, Willnow TE, Christensen EI, Mobley WB, Nykjær A, Andersen OM (2012) Retromer binds the FANSHY sorting motif in SorLA to regulate amyloid precursor protein sorting and processing. J Neurosci 32:1467-1480. CrossRef Medline

Guo Q, Li H, Gaddam SS, Justice NJ, Robertson CS, Zheng H (2012) Amyloid precursor protein revisited: neuron-specific expression and highly stable nature of soluble derivatives. J Biol Chem 287:2437-2445. CrossRef Medline

Haass C (2004) Take five-BACE and the gamma-secretase quartet conduct Alzheimer's amyloid beta-peptide generation. EMBO J 23:483-488. CrossRef Medline

Hu F, Padukkavidana T, Vægter CB, Brady OA, Zheng Y, Mackenzie IR, Feldman HH, Nykjaer A, Strittmatter SM (2010) Sortilin-mediated endocytosis determines levels of the frontotemporal dementia protein, progranulin. Neuron 68:654-667. CrossRef Medline

Jansen P, Giehl K, Nyengaard JR, Teng K, Lioubinski O, Sjoegaard SS, Breiderhoff T, Gotthardt M, Lin F, Eilers A, Petersen CM, Lewin GR, Hempstead BL, Willnow TE, Nykjaer A (2007) Roles for the proneurotrophin receptor sortilin in neuronal development, aging and brain injury. Nat Neurosci 10:1449-1457. CrossRef Medline

Kounnas MZ, Moir RD, Rebeck GW, Bush AI, Argraves WS, Tanzi RE, Hyman BT, Strickland DK (1995) LDL receptor-related protein, a multifunctional ApoE receptor, binds secreted beta-amyloid precursor protein and mediates its degradation. Cell 82:331-340. CrossRef Medline

Lane RF, Raines SM, Steele JW, Ehrlich ME, Lah JA, Small SA, Tanzi RE, Attie AD, Gandy S (2010) Diabetes-associated SorCS1 regulates Alzheimer's amyloid-beta metabolism: evidence for involvement of SorL1 and the retromer complex. J Neurosci 30:13110-13115. CrossRef Medline

Mattson MP, Cheng B, Culwell AR, Esch FS, Lieberburg I, Rydel RE (1993) Evidence for excitoprotective and intraneuronal calcium-regulating roles for secreted forms of the beta-amyloid precursor protein. Neuron 10:243254. CrossRef Medline

Munck Petersen C, Nielsen MS, Jacobsen C, Tauris J, Jacobsen L, Gliemann J, Moestrup SK, Madsen P (1999) Propeptide cleavage conditions sortilin/ neurotensin receptor-3 for ligand binding. EMBO J 18:595-604. CrossRef Medline

Nielsen MS, Madsen P, Christensen EI, Nykjaer A, Gliemann J, Kasper D, Pohlmann R, Petersen CM (2001) The sortilin cytoplasmic tail conveys 
Golgi-endosome transport and binds the VHS domain of the GGA2 sorting protein. EMBO J 20:2180-2190. CrossRef Medline

Nielsen MS, Gustafsen C, Madsen P, Nyengaard JR, Hermey G, Bakke O, Mari M, Schu P, Pohlmann R, Dennes A, Petersen CM (2007) Sorting by the cytoplasmic domain of the amyloid precursor protein binding receptor SorLA. Mol Cell Biol 27:6842-6851. CrossRef Medline

Quistgaard EM, Madsen P, Grøftehauge MK, Nissen P, Petersen CM, Thirup SS (2009) Ligands bind to Sortilin in the tunnel of a ten-bladed betapropeller domain. Nat Struct Mol Biol 16:96-98. CrossRef Medline

Roch JM, Masliah E, Roch-Levecq AC, Sundsmo MP, Otero DA, Veinbergs I, Saitoh T (1994) Increase of synaptic density and memory retention by a peptide representing the trophic domain of the amyloid beta/A4 protein precursor. Proc Natl Acad Sci U S A 91:7450-7454. CrossRef Medline

Rohan de Silva HA, Jen A, Wickenden C, Jen LS, Wilkinson SL, Patel AJ (1997) Cell-specific expression of beta-amyloid precursor protein isoform mRNAs and proteins in neurons and astrocytes. Brain Res Mol Brain Res 47:147-156. Medline
Thornton E, Vink R, Blumbergs PC, Van Den Heuvel C (2006) Soluble amyloid precursor protein alpha reduces neuronal injury and improves functional outcome following diffuse traumatic brain injury in rats. Brain Res 1094:38-46. CrossRef Medline

Ulery PG, Beers J, Mikhailenko I, Tanzi RE, Rebeck GW, Hyman BT, Strickland DK (2000) Modulation of beta-amyloid precursor protein processing by the low density lipoprotein receptor-related protein (LRP). Evidence that LRP contributes to the pathogenesis of Alzheimer's disease. J Biol Chem 275:7410-7415. CrossRef Medline

Vaerman JL, Saussoy P, Ingargiola I (2004) Evaluation of real-time PCR data. J Biol Regul Homeost Agents 18:212-214. Medline

Young-Pearse TL, Chen AC, Chang R, Marquez C, Selkoe DJ (2008) Secreted APP regulates the function of full-length APP in neurite outgrowth through interaction with integrin betal. Neural Dev 3:15. CrossRef Medline 